\title{
Patogenicidade de isolados de Corynespora cassiicola a diferentes espécies de plantas
}

\author{
Ricardo Ribeiro Oliveira ${ }^{1,2}$, João Batista Vida ${ }^{1}$, Dauri José Tessmann ${ }^{1}$, Bárbara de Melo Aguiar ${ }^{1,2}$, Marilda Pereira \\ Caixeta $^{1}$, Antonio Lazarini Barboza ${ }^{1,2}$
}

${ }^{1}$ Universidade Estadual de Maringá, Departamento de Agronomia, Av. Colombo, 5790, 87020-900, Maringá, PR, e-mail: jbvida@uem.br; ${ }^{2}$ Bolsista do $\mathrm{CNPq}$

Parte da dissertação de mestrado do primeiro autor.

Autor para correspondência: João Batista Vida

Data de chegada: 17/08/2005. Aceito para publicação em: 27/03/2007.

\section{RESUMO}

Oliveira, R.R., Vida, J.B., Tessmann, D.J., Aguiar, B.M., Caixeta, M.P, Barboza, A.A.L.. Patogenicidade de isolados de Corynespora cassiicola a diferentes espécies de plantas. Summa Phytopathologica, v.33, n.3, p.297-299, 2007.

Corynespora cassiicola, relatado como um patógeno com ampla gama de espécies hospedeiras, tem causado danos em várias culturas de interesse comercial. Neste trabalho avaliou-se, em ambiente de casa-de-vegetação, a patogenicidade de 15 isolados de C. cassiicola originados de diferentes espécies hospedeiras, quando inoculados em 12 diferentes espécies vegetais. Os isolados de pepino foram os mais patogênicos. Além dos híbridos de pepino, estes infectaram outras seis espécies vegetais testadas. Já os isolados de trapoeraba e de alface foram os que apresentaram menor patogenicidade, pois além dos hospedeiros originais infectaram somente uma espécie hospedeira. A maioria dos isolados apresentou elevada inespecificidade. As espécies vegetais testadas reagiram de formas diferentes quando inoculadas com diferentes isolados. O mamoeiro apresentou maior suscetibilidade, sendo infectado por 12 dos 15 isolados. Contrariamente, a trapoeraba e o assa-peixe foram suscetíveis a três e dois isolados, respectivamente.

Palavras-chave adicionais: mancha alvo, Cucumis sativus.

\section{ABSTRACT}

Oliveira, R.R., Vida, J.B., Tessmann, D.J., Aguiar, B.M., Caixeta, M.P., Barboza, A.A.L.. Pathogenicity of Corynespora cassiicola isolates on different host plants. Summa Phytopathologica, v.33, n.3, p.297-299, 2007.

Corynespora cassiicola, causes target leaf spot on a wide host range, including several economically important crops. This study investigated the pathogenicity of 15 C. cassicola isolates on several hosts, in greenhouse. The fungal isolates were collected from several hosts. These isolates were inoculated on 12 different plant species. The isolates from cucumber showed the widest host range, infecting also six other hosts. The isolates from $C$. benghalensis and lettuce showed the narrowest host range, since they infected their host of origin and only another host plant. Most of the isolates lacked host specificity. Papaya plant, which showed the greatest susceptibility to the C. cassiicola isolates, were colonized by 12 of the 15 isolates tested. On the opposite, Vernonia sp. and $C$ benghalensis were susceptible to only two and three C. cassiicola isolates, respectively.

Additional Keywords: target leaf spot, Cucumis sativus.

Corynespora cassiicola (Berk. \& Curt.) Wei, causa doença em mais de 70 espécies de hospedeiros distribuídos em diversos países de clima tropical e subtropical (8). Ellis (3) descreveu C. cassiicola como sendo uma espécie cosmopolita e inespecífica, comum e abundante em regiões tropicais.

No Brasil, o patógeno já foi relatado causando danos em diversas espécies de plantas, tais como: soja (11), pepino (10), tomateiro (4), mamoeiro e cacaueiro (2), alface (Lactuca sativa) (informação verbal fornecida pelo Dr. Luiz Sebastião Poltronieri em novembro de 2003), entre outras. Também é citado como patógeno em algumas plantas daninhas, tais como, trapoeraba (Commelina benghalensis) e assapeixe (Vernonia cinerea) (9).

A capacidade de $C$. cassiicola infectar diferentes espécies vegetais evidencia diferenças de patogenicidade nas populações dentro da espécie. Essas diferenças foram utilizadas por Olive et al. (5) para a proposição de duas raças para a espécie, baseando-se nos tipos de lesões produzidas nos hospedeiros: a raça 1 isolada de feijão-de-corda e a raça 2 de soja. Cutrim \& Silva (1) constataram que dois isolados de C. cassiicola, originados de folhas de tomateiro, apresentaram alta variabilidade na patogenicidade, quando inoculados em várias espécies de plantas agronômicas e daninhas.

Visto a ampla gama de hospedeiras de C. cassiicola e a sua capacidade em infectar hospedeiros comuns e distintos, estudos para se determinar a capacidade de isolados do patógeno em infectar diferentes plantas hospedeiras passa a ser de grande importância. Assim o presente trabalho teve como objetivo verificar a patogenicidade de 15 isolados de C. cassiicola obtidos de diferentes espécies vegetais em causar doença em outras espécies.

O ensaio experimental foi realizado em casa-de-vegetação e o delineamento experimental foi inteiramente casualizado, com seis 
repetições para cada tratamento, sendo uma planta considerada uma unidade experimental.

Três isolados foram obtidos de plantas de pepino através de isolamentos a partir de folhas. Os outros 12 isolados foram cedidos por centros de ensino e pesquisa. Todos os isolados utilizados nos experimentos (Tabela 1) partiram de culturas monospóricas. Para a obtenção destas culturas, uma suspensão de esporos foi plaqueada em ágar-água $(2 \%)$, posteriormente, conídios germinados foram transferidos individualmente para placas contendo meio BDA. Todos os isolados foram acondicionadas em tubos de ensaio e armazenados em geladeira a $8 \pm 2^{\circ} \mathrm{C}$. As espécies de plantas utilizadas neste trabalho estão descritas na Tabela 1.

Teste de patogenicidade: As mudas foram produzidas em bandejas de isopor com substrato comercial e posteriormente foram transplantadas para sacos de plástico de capacidade de $1 \mathrm{~L}$ contendo substrato solo:areia na proporção 1:1, desinfestado com brometo-demetila visando eliminar outros microorganismos.

O inóculo foi produzido em meio BDA e as suspensões de conídios para serem inoculados foram ajustadas para a concentração de $1 \times 10^{4}$ esporos. $\mathrm{mL}^{-1}$ utilizando um hemacitômetro. Para a inoculação foi utilizado um atomizador, De Vilbs $n^{\circ} 15$. O inóculo foi aspergido sobre as folhas até ao "ponto de escorrimento" e em ambas as faces, no estádio em que as espécies vegetais apresentavam o número de folhas suficiente para a inoculação (Tabela 1). Após a inoculação, as plantas foram incubadas em câmara úmida por 48 horas e em seguida retornadas à condição de casa-de-vegetação.

A reação foi considerada positiva (suscetível) ou negativa (imune) em função da presença ou ausência de sintomas da doença e de sinais do patógeno nos limbos foliares das espécies testadas. Os resultados do experimento estão expressos na Tabela 2.

A maioria dos isolados apresentou elevada inespecificidade para os hospedeiros e o tempo para aparecimento de sintomas variou entre as espécies de plantas. Em híbridos de pepino inoculados com os isolados IA, PB, JQ e LP05 e em soja inoculada com os isolados 493AA e 777AA, os sintomas se manifestaram dentro de 48 horas. Já para as demais espécies, os sintomas se manifestaram 96 horas após a inoculação.

Os isolados de pepino foram os mais patogênicos infectando todas as espécies hospedeiras, exceto trapoeraba e assa-peixe, seguido do isolado de abóbora que infectou nove das 12 espécies hospedeiras inoculadas. Por outro lado, os isolados de alface e trapoeraba infectaram apenas duas das 12 espécies inoculadas.

Os isolados IA, PB e JQ de pepino e o isolado LP05 de abóbora foram patogênicos aos híbridos de pepino 'Natsubayashi', 'Hokushin', 'Tsuyataro' e 'Samurai', ao híbrido 'Sunrise' de mamoeiro, ao híbrido 'Sunrise' de meloeiro nobre, à variedade 'Exposição' de abóbora, a cultivar 'FT-estrela' de soja e à variedade 'Santa Clara' de tomateiro. Além disso, os três isolados de pepino também infectaram plantas da variedade 'Vera' de alface. Estes isolados se comportaram semelhantemente quanto à capacidade de causar doenças aos diferentes hospedeiros testados.

Diferentemente, os isolados 493AA e 777AA de soja apresentaram diferenças quanto a patogenicidade. $\mathrm{O}$ isolado 493AA foi patogênico ao mamoeiro, a dois híbridos de pepino, 'Natsubayashi' e 'Tsuyataro', ao meloeiro, à abóbora, à soja, à alface e ao tomateiro. Já, o isolado 777AA foi patogênico a dois híbridos de pepino ('Natsubayashi' e 'Tsuyataro'), à soja, à alface e ao tomateiro. Todas as espécies suscetíveis ao isolado 777AA também o foram ao isolado 493AA.

Além do tomateiro, o isolado LP02 foi patogênico a dois híbridos de pepino ('Natsubayashi' e 'Tsuyataro') e ao mamoeiro. Os isolados GS01 e RWB321 de mamoeiro e falso-boldo, respectivamente, além do mamoeiro, também infectaram o meloeiro nobre, a soja e a alface, mostrando serem similares entre si quanto à capacidade de causar doenças a diferentes espécies de plantas.

O isolado de L. camara (JMP220), foi patogênico ao mamoeiro, à alface, ao tomateiro e ao assa-peixe. O isolado de aceroleira (LP04) infectou o meloeiro nobre, abóbora, soja e a trapoeraba. $\mathrm{O}$ isolado de alface (LP01) foi patogênico a alface e ao tomateiro. $\mathrm{O}$ isolado de trapoeraba (CP03), além da trapoeraba, foi patogênico a abóbora. Estes isolados, LP01 e CP03, foram os que apresentaram a menor gama de hospedeiros dentre os isolados avaliados. O isolado LP07 de pimenta-longa foi patogênico ao mamoeiro, a abóbora e ao tomateiro. O isolado de assa-peixe (GS02), além de assa-peixe, foi patogênico ao mamoeiro, à soja, à alface e a trapoeraba. $\mathrm{O}$ mamoeiro foi o hospedeiro que apresentou maior suscetibilidade frente aos isolados testados, sendo infectado por 12 dos 15 isolados. Ao contrário, a trapoeraba e o assa-peixe foram os menos suscetíveis, sendo infectados por três e dois isolados de C. cassiicola, respectivamente.

A inespecificidade de isolados de C. cassiicola constatada neste trabalho foi também observada por outros autores. Duarte et al. (2) observaram que um isolado de mamoeiro infectava plantas de

Tabela 1. Isolados de Corynespora cassiicola, sua procedência e espécies hospedeiras utilizadas para inoculação. Maringá, PR., 2005

\begin{tabular}{|c|c|c|c|c|c|}
\hline \multirow{2}{*}{ Isolado } & \multirow[b]{2}{*}{ Hospedeiro de origem } & \multicolumn{2}{|c|}{ Procedência } & \multicolumn{2}{|c|}{ Hospedeiro inoculado } \\
\hline & & Estado & Espécie & Idade(dias) & $\mathrm{N}^{0}$ de folhadefinitiva \\
\hline IA & Pepino & Paraná & Pepino/hokushin & 16 & 2 \\
\hline $\mathrm{PB}$ & Pepino & Paraná & Pepino/natsubayashi & 16 & 2 \\
\hline JQ & Pepino & Paraná & Pepino/tsuyataro & 16 & 2 \\
\hline 493AA & Soja & Paraná & Pepino/samurai & 16 & 2 \\
\hline 777AA & Soja & Paraná & Melão/sunrise & 15 & 2 \\
\hline JMP220 & Lantana camara & Minas Gerais & Abóbora/exposição & 15 & 2 \\
\hline $\mathrm{CP} 03$ & Commelina benghalensis(trapoeraba) & Minas Gerais & Soja/FT-estrela & 22 & 3 \\
\hline RWB321 & Coleus barbatus & Minas Gerais & Alface/vera & 20 & 7 \\
\hline GS01 & Mamoeiro & Maranhão & Tomateiro/santaclara & 25 & 4 \\
\hline GS02 & Vernonia sp. (assa-peixe) & Maranhão & Mamoeiro/sunrise & 30 & 5 \\
\hline LP0 1 & Alface & Amazonas & Trapoeraba & 25 & 3 \\
\hline LP02 & Tomateiro & Amazonas & Assa-peixe & 35 & 4 \\
\hline LP04 & Malpighia gabra(aceroleira) & Amazonas & & & \\
\hline LP05 & Abóbora & Amazonas & & & \\
\hline LP07 & Pipper hispidinervium (pimenta longa) & Amazonas & & & \\
\hline
\end{tabular}


Tabela 2. Patogenicidade de isolados de Corynespora cassiicola originados de diferentes hospedeiros inoculados à temperatura ambiente em casa de vegetação. UEM, Maringá, PR., 2005

\begin{tabular}{|c|c|c|c|c|c|c|c|c|c|c|c|c|c|c|c|}
\hline \multirow[b]{2}{*}{ Espécie vegetal } & \multicolumn{15}{|c|}{ Código do isolado e hospedeiro } \\
\hline & $\begin{array}{c}\text { IA } \\
\text { Pepino }\end{array}$ & $\begin{array}{c}\text { PB } \\
\text { Pepino }\end{array}$ & $\begin{array}{c}\text { JQ } \\
\text { Pepino }\end{array}$ & $\begin{array}{c}\text { LP05 } \\
\text { Abóbora }\end{array}$ & $\begin{array}{l}\text { 493AA } \\
\text { Soja }\end{array}$ & $\begin{array}{l}\text { 777AA } \\
\text { Soja }\end{array}$ & $\begin{array}{c}\text { LP02 } \\
\text { Tomateiro }\end{array}$ & $\begin{array}{c}\text { GS01 } \\
\text { Mamoeiro }\end{array}$ & $\begin{array}{l}\text { JMP220 } \\
\text { Lantana }\end{array}$ & $\begin{array}{c}\text { LP04 } \\
\text { Aceroleira }\end{array}$ & $\begin{array}{l}\text { LP01 } \\
\text { Alface }\end{array}$ & $\begin{array}{c}\text { LP07 } \\
\text { Pimenta-longa }\end{array}$ & $\begin{array}{c}\text { RWB321 } \\
\text { Falso-boldo }\end{array}$ & $\begin{array}{c}\text { CP03 } \\
\text { Trapoeraba }\end{array}$ & $\begin{array}{l}\text { Assa-peixe } \\
\text { GS02 }\end{array}$ \\
\hline $\begin{array}{l}\text { Carica papaya } \\
\text { "Sunrise" }\end{array}$ & $+{ }^{1}$ & + & + & + & + & - & + & + & + & + & - & + & + & - & + \\
\hline $\begin{array}{l}\text { Cucumis sativus } \\
\text { "Hokushin" }\end{array}$ & + & + & + & + & - & - & - & - & - & - & - & - & - & - & - \\
\hline $\begin{array}{l}\text { Cucumis sativus } \\
\text { "Natsubayashi" }\end{array}$ & + & + & + & + & + & + & + & - & - & - & - & - & - & - & - \\
\hline $\begin{array}{l}\text { Cucumis sativus } \\
\text { "Samurai" }\end{array}$ & + & + & + & + & - & - & - & - & - & - & - & - & - & - & - \\
\hline $\begin{array}{l}\text { Cucumis sativus } \\
\text { "Tsuyataro" }\end{array}$ & + & + & + & + & + & + & + & - & - & - & - & - & - & - & - \\
\hline $\begin{array}{l}\text { Cucumis melo } \\
\text { "Sunrise" }\end{array}$ & + & + & + & + & + & - & - & + & - & + & - & - & + & - & - \\
\hline $\begin{array}{l}\text { Cucurbita maxima } \\
\text { "Exposição" }\end{array}$ & + & + & + & + & + & - & - & - & - & + & - & + & - & + & - \\
\hline $\begin{array}{l}\text { Glycine Max } \\
\text { "FT-estrela" }\end{array}$ & + & + & + & + & + & + & - & + & - & + & - & - & + & - & + \\
\hline $\begin{array}{l}\text { Lactuca sativa } \\
\text { "Vera" }\end{array}$ & + & + & + & - & + & + & - & + & + & - & + & - & + & - & + \\
\hline $\begin{array}{l}\text { Lycopersicon esculentum } \\
\text { "Santa Clara" }\end{array}$ & + & + & + & + & + & + & + & - & + & - & + & + & - & - & - \\
\hline $\begin{array}{l}\text { Commelina } \\
\text { benghalensis }\end{array}$ & - & - & - & - & - & - & - & - & - & + & - & - & - & + & + \\
\hline Vernonia sp. & - & - & - & - & - & - & - & - & + & - & - & - & - & - & + \\
\hline
\end{tabular}

$(+)$ presença de sintomas e sinais do patógeno, (-) ausência de sintomatologia

mamoeiro, caupi e seringueira, já um isolado originado de cacaueiro era específico para plantas de cacaueiro.

Cutrim \& Silva (1) relataram diferenças na patogenicidade de dois isolados de tomateiro. Estes apresentaram comportamentos diferentes quando inoculados em plantas de caupi (isolado 1 não patogênico e isolado 2 patogênico) e soja (isolado 2 não patogênico e isolado 1 patogênico). Além disso, estes isolados foram patogênicos a outras oito espécies vegetais.

Silva et al. (7) encontraram reações semelhantes às acima relatadas, onde 16 isolados de seringueira, três de mamoeiro, um de mimosa (Mimosa invisa) e um de tomilho (Thymus vulgaris) diferiram quanto à patogenicidade, quando inoculados em plantas de berinjela e de feijãode-corda.

Roim (6) usou o termo adaptação ao relatar que isolados originados das raízes de soja foram mais agressivos do que os originados das folhas, quando inoculados em raízes de soja. O contrário também foi observado. O autor ainda sugere a possibilidade da existência de diferentes biótipos ou espécies de Corynespora infectando raízes e folhas de soja.

Dentro da espécie C. cassiicola, isolados originados de diferentes hospedeiros podem se diferenciar quanto à patogenicidade, sendo que alguns podem ter maior ou menor gama de hospedeiros. Um isolado de C. cassiicola originado de uma determinada espécie de planta não necessariamente será capaz de infectar uma outra espécie de planta também reconhecida como hospedeira do patógeno.

Como constatado, diferentes espécies de plantas podem ser infectadas por isolados de $C$. corynespora, que pode causar danos significativos em espécies vegetais de importância agronômica $(4,10)$. As hospedeiras podem ainda se constituir em importante fonte de inóculo para outras culturas (1). Assim, informações sobre o patossistema hospedeiro/C. cassiicola vem a ser importantes para o manejo da doença.

\section{REFERÊNCIAS BIBLIOGRÁFICAS}

1- Cutrim, F.A.; Silva, G.S. Patogenicidade de Corynespora cassiicola a diferentes espécies de plantas. Fitopatologia Brasileira, Brasília, v.28, n.2, p.193-194, 2003.

2- Duarte, M.L.R.; Asano, S.; Albuquerque, F.C. Estudos comparativos das características morfológicas e fisiológicas de dois isolamentos de Corynespora cassiicola. Fitopatologia Brasileira, Brasília, v.8, n.2, p.205-214, 1983.

3- Ellis, M.B. Dematiaceous hyphomycetes. Kew: Commonwealth Mycological Institute, 1971. 608p.

4- Leroy, M.; Lourd, M. Doença foliar do tomateiro causada por Corynespora cassiicola em Manaus. Fitopatologia Brasileira, Brasília, v.14, n.1, p.32-36, 1989.

5- Olive, L.S.; Bain, D.C.; Lefebvre, C.L. A leaf spot of cowpea and soybean caused by undescribed species of Helminthosporium. Phytopathology, St. Paul, v.35, n.5, p.822-831, 1945.

6- Roim, F.L.B. Comportamento de cultivares e caracterização cultural, morfologia e patogênica de isolados de Corynespora cassiicola obtidos de soja [Glycine max (L.) Merrill]. 2001. 72f. Dissertação (Mestrado em Agronomia) - Faculdade de Ciências Agrárias e Veterinárias, Universidade Estadual Paulista, Jaboticabal.

7- Silva, W.P.K.; Deverall, B.J.; Lyon, B.R. Molecular, physiological and pathological characterization of Corynespora leaf spot fungi from rubber in Sri Lanka. Plant Pathology, Oxford, v.47, n.2, p.267-277, 1998.

8- Silva, W.P.K.; Multani, D.S.; Deverall, B.J.; Lyon, B.R. RFLP and RAPD analyses in the identification and differentiation of isolates of the leaf spot fungus Corynespora cassiicola. Australian Journal of Botany, Melbourne, v.43, n.3, p.609-618, 1995.

9- Souza, I.M.R.; Silva, G.S. Fungos associados a plantas daninhas na ilha de São Luiz, Maranhão. Summa Phytopathologica, Botucatu, v.27, n.2, p.267-268, 2001.

10- Verzignassi, J.R.; Vida, J.B.; Tessmam, D.J. Corynespora cassiicola causando epidemias de manchas foliares em pepino "japonês" sob estufa no norte do Paraná. Fitopatologia Brasileira, Brasília, v.28, n.5, p.570, 2003.

11- Yorinori, J.T.; Homechin, M. Doenças de soja identificadas no Estado do Paraná no período de 1971 a 1976. Fitopatologia Brasileira, Brasília, v.2, n.1, p.108, 1977. 